\title{
Lupus mimickers...!
}

\section{Howaida E. Mansour, Nahla Hussin, Rahma Elziaty, Warda Abdelfattah}

Department of Internal Medicine - Rheumatology Division, Ain Shams University; Egypt

A 51 years old female single, born and live in Qena, have 20 years history of extensive skin rash involving the whole body including the face, in the form of background erythema with hyper pigmented areas alternating with de pigmented areas and yellow islands.

According to her description - it is sometimes very itchy, she sought medical advice diagnosed as rheumatoid arthritis and has been described DMARDs therapy but this was of no value.
Three years ago there was super added alopecia and progressive arthralgias involving almost all joints including the wrist and the small joints of the hands, occasionally there is fevere and weight loss. Examination was unremarkable except of $2 \times 3 \mathrm{~cm}$ axillary lymphadenopathy.

ESR 50, CRP 24, HB 9 gm\%, ANA + ve $1 / 80$ speckled, DNA +ve 42

Urine analysis is free and $\mathrm{P} / \mathrm{C}$ ratio is normal.
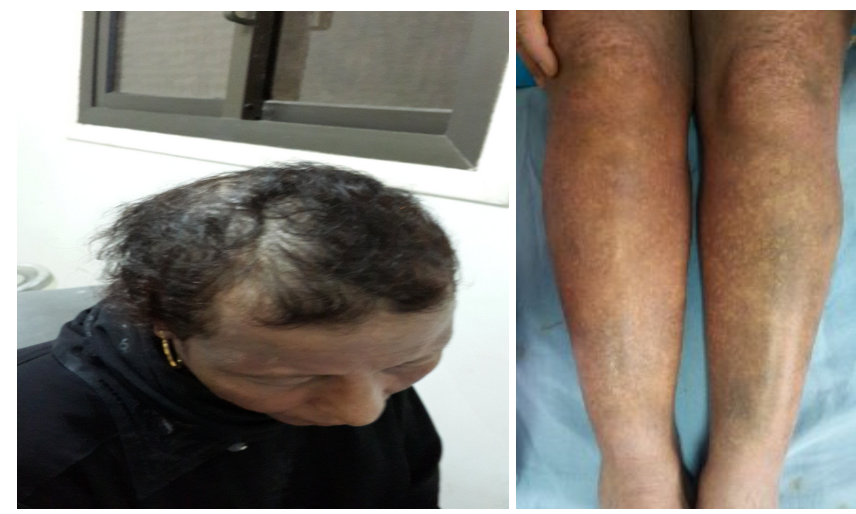

Figure 1. Alopecia and generalized skin rash.
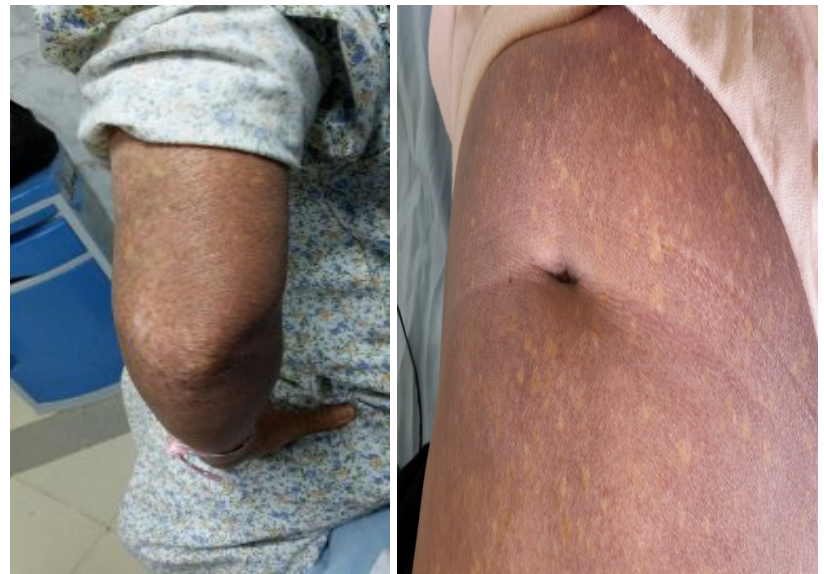

Figure 2. Diffuse skin rash involving the whole body.

Correspondence to Howaida E. Mansour,

e-mail: howaida_mansour@hotmail.com 


\section{Discussion}

Clinically this lady is fulfilling the SLICC classification criteria for $\mathrm{SLE}^{1}$ so we put the provisional diagnosis to be one of the following:

- SLE with extensive unusual lupus rash

- Subacute cutaneous lupus

- $\quad$ Skin psoriasis with psoriatic arthritis

- $\quad$ Others..??

Dermatological consultation with full punch skin biopsy and histopathology was done:

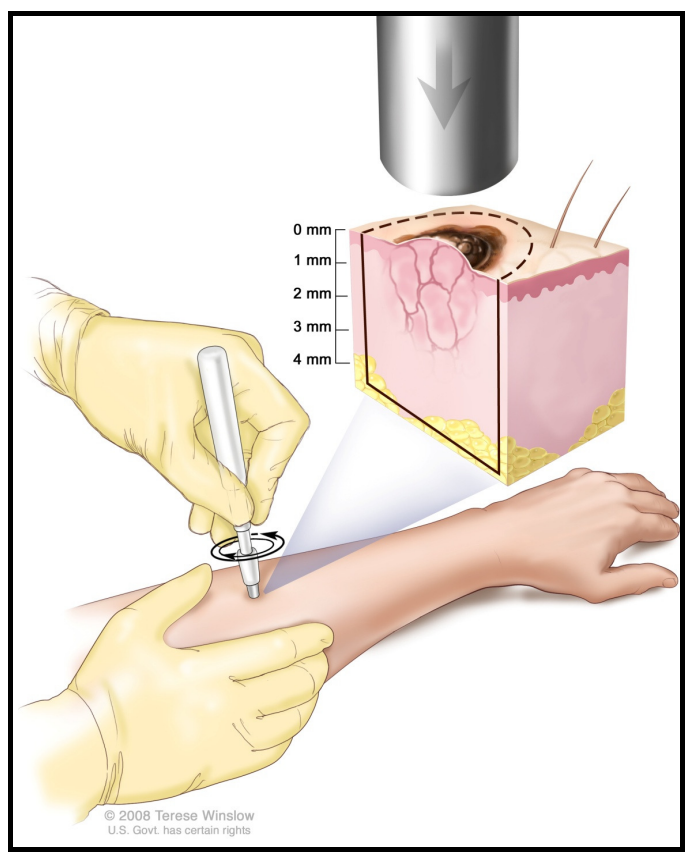

Figure (3)

It reveals upper dermal heavy infiltration by chronic inflammatory cells with scattered atypical hyperchromatic lymphocytes are seen infiltrating the lumen of the epidermis, picture is compatible with Erythroderma mycosis fungoid.

Erythroderma mycosis fungoid (MF), is a rare type of T cell Non Hodgkin lymphoma (NHL) of the skin, that clinically closely mimic SLE. Interestingly this type of skin lymphoma causes diffuse alopecia, erythematous skin rash, arthralgias, constitutional symptoms and positive ANA/anti-DNA testing...!!

Serial CT scans with contrast was done on the neck, chest and abdomen was done for staging of the lymphoma; it shows two cervical and multiple inguinal lymphadenopathy.
Typically the natural history of MF is indolent and the prognosis depends largely on the extent of skin involvement at presentation. The disease manifestations may present over years up to 20 years as waxing and waning cutaneous eruption ${ }^{3}$.

The cutaneous disease typically progresses from an eczematous patch/plaque stage covering less than $10 \%$ of the body surface (T1) to plaque stage covering $10 \%$ or more of the body surface (T2), and finally to tumors (T3) that frequently undergo necrotic ulceration $^{2,4}$. A retrospective study with a median follow-up for 14.5 years showed that $20 \%$ of the 1,422 patients progressed from stage I or II disease to stage III or IV disease ${ }^{5}$.

Sezari syndrome (SS) represents generalized erythroderma mycosis fungoid stage (T4) with peripheral blood involvement with atypical lymphocytes. However, there is some disagreement about whether the MF and SS are actually variants of the same disease ${ }^{6}$. The patient was referred to hematology department to start chemotherapy protocol for NHL lymphoma.

\section{References}

1. Petri M, Orabi AM,Alarcon GS et al: Drivation and validation of systemic lupus international collaborating clinics classification criteria of systemic lupus erythematosus. Arthritis Rheum; 64:2677, 2012.

2. Siegel RS, Pandolfino T, Guitart J, et al.: Primary cutaneous T-cell lymphoma: review and current concepts. J Clin Oncol 18 (15): 2908-25, 2000.

3. Diamandidou E, Cohen PR, Kurzrock R: Mycosis fungoides and Sezary syndrome. Blood 88 (7): 2385-409, 1996

4. Lorincz AL: Cutaneous T-cell lymphoma (mycosis fungoides) Lancet 347 (9005): 871-6, 1996.

5. Quaglino P, Pimpinelli N, Berti E, et al.: Time course, clinical pathways, and long-term hazards risk trends of disease progression in patients with classic mycosis fungoides: a multicenter, retrospective follow-up study from the Italian Group of Cutaneous Lymphomas. Cancer 118 (23): 5830-9, 2012.

6. Olsen EA, Rook AH, Zic J, et al.: Sézary syndrome: immunopathogenesis, literature review of therapeutic options, and recommendations for therapy by the United States Cutaneous Lymphoma Consortium (USCLC). J Am Acad Dermatol 64 (2): 352 - 404, 2011. 